\title{
ACTORES EDUCATIVOS: DIVERGENCIAS EN LOS CANALES DE COMUNICACIÓN Y SUS EFECTOS EN LA GESTIÓN DE LA I.E.I. No 0325-RÍMAC.
}

\author{
EDUCATIONAL ACTORS: DIVERGENCES IN THE COMMUNICATION CHANNELS AND THEIR \\ EFFECTS IN THE MANAGEMENT OF THE I.E.E. N. ${ }^{\circ}$ 325-RIMAC.
}

Adela Victoria Hinostroza Bruno ${ }^{1}$

\begin{abstract}
RESUMEN
La Institución Educativa Inicial No 0325 cumplirá 41 años de funcionamiento. En la actualidad cuenta con 56 niños y niñas matriculados. El personal está formado por 5 personas: tres docentes, una auxiliar y una trabajadora que se encarga de la limpieza. Desde el año 2008, el alumnado de esta institución disminuyó considerable y paulatinamente (de 80 a 40 estudiantes). Por lo cual, corre el riesgo de ser cerrada. El presente artículo es un diagnóstico de esta situación. Por eso, describe y analiza las divergencias en la comunicación entre los actores educativos de esta institución. Propone la provisión de estrategias tendientes a desarrollar la competencia comunicativa de los actores para que la institución tenga una mejor gestión: no haya deserción estudiantily, en última instancia, no sea cerrada.
\end{abstract}

Palabras clave: Actores educativos; Comunicación horizontal; Comunicación jerárquica; Promoción escolar, Deserción escolar

\section{ABSTRACT}

The Initial Educational Institution No. 325 will be 41 years old. At present it has 56 children enrolled. The staff consists of 5 people: three teachers, an assistant and a worker who is responsible for cleaning. Since 2008, the students of this institution decreased considerably and gradually (from 80 to 40 students). Therefore, it runs the risk of being closed. This article is a diagnosis of this situation. Therefore, it describes and analyzes the divergences in communication between the educational actors of this institution. It proposes the provision of strategies tending to develop the communicative competence of the actors so that the institution has a better management: there is no student desertion and, ultimately, it is not closed.

Key words: Educational actors; Horizontal communication; Hierarchical communication; School promotion; School dropout

1 Universidad Nacional José Faustino Sánchez Carrión (Huacho - Perú). Email: vinvestigación@unjfsc.edu.pe 


\section{INTRODUCCIÓN}

El proceso de enseñanza aprendizaje no vincula únicamente a docentes y alumnos, sino se trata de un proceso en donde intervienen todas las personas que laboran en la institución educativa, además de los tutores y madres/padres de familia. A todas estas personas se les denomina actores educativos, porque van a influir de una u otra manera en el éxito de la formación académica de los niños y niñas. Entre estos actores debe haber una comunicación constante, que sea asertiva y propulse los logros de aprendizaje propuestos. ¿Qué ocurre cuando esta comunicación falla? ¿Cuándo decimos que hay divergencias en los canales de comunicación de una institución educativa? ¿Cómo se puede mejorar la comunicación entre los actores educativos?

Este es el tema del presente artículo. La institución educativa $\mathrm{N}^{\circ}$ 0325-Rímac para el nivel inicial se centra principalmente en la atención y formación de los niños y niñas de una forma tradicional, es decir, con temas generadores de contenidos educativos y dirigidos por las docentes, hojas de aplicación y la memoria como factor principal del proceso de aprendizaje. No se da opción de participación a los padres quizás porque no se sabe qué estrategia emplear para incluirlos y las relaciones con ellos varían según la actitud de la docente, lo cual ha venido generando un clima de insatisfacción entre los padres, quienes al observar la conducta y ejercicio profesional de las docentes han creado simpatías y antipatías con las mismas. Esto último ha ocasionado el retiro de alumnado de la institución y que, año tras año, la oferta educativa en el sector haga menos atractiva a la I. E. I. N 0325 que corre, incluso, el riesgo de ser cerrada por el poco alumnado que se matricula cada año.

A partir de la evaluación que hubo el año 2017 y con la advertencia de la especialista de los problemas que afronta la institución, se está trabajando en mostrar otra imagen a nivel social y educativo. Se está dialogando con los padres y madres de familia para el cambio de nombre de la institución y se está trabajando con las rúbricas propuestas por el Ministerio de Educación para aplicarlos en el proceso de enseñanza aprendizaje en este nivel. Sin embargo, es notorio que faltan estrategias o herramientas para procesar y solucionar los conflictos en la escuela. No basta la buena voluntad de los actores educativos.

Por consiguiente, con este estudio se busca contribuir a mejorar los canales de comunicación entre los actores educativos de la I. E. I. N ${ }^{\mathrm{o}} 0325$ para la organización interna entre actores, resolución de conflictos internos y externos y la generación de un clima propicio para los procesos de enseñanza aprendizajes. Así, se pretende promover el diálogo entre todos los actores educativos y eliminar las brechas que frenan el desarrollo de la institución y su reconocimiento en la sociedad después de 40 años al servicio de la comunidad.

\section{Ubicación geográfica y aspectos sociales}

La I. E. I. N $N^{0} 0325$ tiene 40 años de creación, funciona como colegio de transición, primer y segundo grado de primaria. El tránsito peatonal por alrededor de la Institución es muy fluido. Se encuentra ubicada en la Av. Tomas Vidal sin número, a una cuadra de la avenida Tarapacá, en una zona conocida como La Totorita. Es más así se le conoce a esta institución, lo cual ha repercutido negativamente en la visión que se tiene de este centro ya que este sector es reconocido por las propias autoridades municipales y policiales como una zona peligrosa, donde residen algunas personas de mal vivir, involucradas en prácticas delincuenciales como robo, micro comercialización de drogas, etc. Además de generar disturbios como peleas callejeras por el consumo de licor en las calles. Así, el nombre Las Totoritas connota estas prácticas delincuenciales, a pesar de que, en la actualidad, han disminuido estos actos porque hay más vigilancia en el sector. Se desarrollan diversas actividades económicas como polladas, campeonatos de fulbito.

Esta institución educativa no es la única en el sector. Está rodeada de colegios privados y públicos. A dos cuadras cruzando la pista de la avenida Tarapacá se encuentra La Gran Unidad Emblemática Ricardo Bentín; a 5 cuadras, el colegio privado Pestalozzi y el colegio Ramón Castilla que atiende PRONOEI, Inicial, primaria y secundaria. Entonces, la oferta educativa en la zona es amplia. Los padres de familia tienen libertad para elegir el centro en donde sus hijos van a estudiar.

\section{Descripción de los actores educativos}

Se denomina actores educativos a quienes intervienen directa o indirectamente en los procesos de enseñanza aprendizaje de los niños y están articulados en torno a la escuela como institución forjadora de nuevos conocimientos y de socialización. No solo se trata de profesionales de la educación, sino de todas aquellas personas que influyen en el niño de alguna u otra manera. Por eso, se considera que los actores educativos no solo están 
dentro de la escuela, sino también fuera de ella, específicamente en el hogar.

Los actores son considerados agentes que operan como ejemplo de conductas, guía, orientadores del proceso de aprendizaje de los niños. En ese sentido, los problemas educativos a los que estos se enfrenten deben ser resueltos de forma colaborativa. Para esto es muy importante la comunicación; por eso, existe "en los últimos años cierta preocupación por intervenir en la calidad de las interacciones de los actores del sistema educativo, como condicionante de una gestión de calidad hacia la consecución de los deseados resultados de aprendizaje" (Murillo y Becerra, 2009, p. 378).

\subsection{Directora}

Según la Ley General de Educación 28044, Art. 53 (2003), el "director es la máxima autoridad y el representante legal de la Institución Educativa. Es responsable de la gestión en los ámbitos pedagógico, institucional y administrativo". Entre sus principales funciones, al director le corresponde cumplir con lo dispuesto en la Ley en materia a lo que su función como gestor implica. El director es el principal promotor de las relaciones sociales entre los actores educativos, es el líder en la institución y modelo de profesional. Como tal, debe promover la transparencia de su gestión a través de la evaluación y autoevaluación y el conocimiento de estos resultados a la comunidad.

La I. E. I. N $N^{\circ} 0325$ está a cargo de una directora quien además ejerce la docencia y está a cargo del aula de niños de 5 años. Bajo su gestión, estamos las docentes de dos grupos: las aulas con alumnos de 3 y 4 años, una auxiliar y la señora de limpieza. Nuestro trabajo consiste en la atención a niños de 3 a 5 años quiere decir el II Ciclo de la EBR, preparación básica para potenciar su desarrollo cognitivo, emocional y físico utilizando el juego como nuestra estrategia principal para estos aprendizajes.

La profesora Zoraida Altamirano de 57 años de edad, directora de la I. E. I. No 0325 ocupa el cargo desde el año 2017, por concurso del Ministerio de Educación. Estará dirigiendo el centro por 4 años consecutivos. Al parecer, que tenga a su cargo un aula además del cargo de directora, dificulta que organice a todos los actores educativos y monitoree nuestras clases. A pesar de su figura de líder en la institución, ha generado conflictos internos porque, principalmente, la comunicación con los demás actores no es la mejor. Los conflictos se originan en la rendición de cuentas a los padres de familia.
Definitivamente, es entre directores y padres de familia que no se logra una comunicación eficaz.

\subsection{Profesoras}

En Ley General de Educación 28044, Art. 54, se conceptualiza el rol del profesor, quien es visto como un "agente fundamental del proceso educativo y tiene como misión contribuir eficazmente en la formación de los estudiantes en todas las dimensiones del desarrollo humano". El profesor o profesora no solo es un profesional idóneo, sino una persona proba de demostrada salud física y mental. Sus principales funciones además de planificar, desarrollar y evaluar actividades que aseguren el logro del aprendizaje de los estudiantes, son cumplir las normas institucionales de convivencia en la comunidad educativa que integran y participar en la institución educativa y en otras instancias a fin de contribuir al desarrollo del Proyecto Educativo Institucional, así como del Proyecto Educativo Local, Regional y Nacional.

La I. E. I. N 0325 tiene tres profesoras, pero una ocupa además el cargo de directora, quien es responsable de los niños de 5 años de edad y tiene en su aula 15 niños. Generalmente, asisten solo 10 niños. Su trabajo de docente es percibido como parco por los padres de familia. Incluso, se le acusa de ser fría y distante con los niños. Al inicio del año escolar, pidió a las demás profesoras no darles besos a los niños por los problemas que pueden surgir con el tema de alguna denuncia por acercamiento del niño. Las muestras de afecto son un tema cultural. Al menos entre las profesoras y los alumnos se crea un clima de mucha expresividad donde los afectos se muestran de manera espontánea y son los niños quienes buscan y reclaman ese afecto y acercamiento con sus profesoras.

La Profesora Delia Figueroa de 45 años de edad, personal nombrado, tiene a su cargo el aula de 4 años con 20 niños, de los cuales asisten alrededor de 15 niños. La profesora tiene 19 años en la Institución, estuvo como Directora encargada en dos oportunidades. Por el compromiso de la Evaluación del Desempeño que se inició el año 2017, obtuvo un puntaje aprobatorio en la Evaluación de Desempeño de las Rubricas del Ministerio de Educación.

La investigadora, profesora Adela Hinostroza de 47 años, viene desempeñándose por tercer año consecutivo en esta institución con el aula de 3 años. Trabaja con los padres de familia usando incluso las redes sociales para informarles y recibir consultas sobre el rendimiento y conducta de los niños. Por 
eso, quizás, se ha ganado la confianza y seguridad para una mejora siguiendo los lineamientos de las capacitaciones. Obtuvo un puntaje alto en la Evaluación de Desempeño de las Rubricas del Ministerio de Educación durante el 2017. para una mejora, siguiendo los lineamientos de las capacitaciones. Obtuvo un puntaje alto en la Evaluación de Desempeño de las Rubricas del Ministerio de Educación durante el 2017.

\subsection{Auxiliar}

En el Decreto Supremo No 008-2014-MINEDU, se establecen las competencias del personal auxiliar de una institución educativa. La principal es apoyar al docente en las actividades formativas y de atención a los niños y niñas en respuesta a sus necesidades de alimentación, fisiológicas, de recreación, salud, aseo y cuidado. Debe informar y coordinar permanentemente con el docente de aula, sobre las observaciones a niños y niñas que requieran una atención especial, como también la de los niños y niñas con necesidades educativas especiales. Con los padres, se vincula al recibir y despedir a los niños de la institución y cuando apoya al docente en las convocatorias y reuniones de padre de familia.

En la I. E. I. N ${ }^{\circ} 0325$, la auxiliar es una señora de 63 años de edad, personal nombrado que labora 30 años en la Institución. Cumple el rol fundamental de apoyar a la Directora en lo que se le solicite, debido a que la Directora tiene aula a cargo. Interviene en el avance de la Institución al estar presente en todo evento que nos compete.

\subsection{Personal de servicio}

El Art. 25: de la Ley General de Educación establece que las funciones del personal de servicio de limpieza son el mantenimiento conservación y limpieza de los ambientes asignados de la I.E, pero además realizar labores de seguridad, vigilancia y portería del centro educativo en su respectivo turno. Por eso, controla el ingreso y salida del alumnado de acuerdo al horario establecido. Además debe controlar y orientar el ingreso y salida del público en general revisando paquetes, maletines, etc. pidiendo obligatoriamente su DNI. para su identificación. También apoya en las tareas de distribución de documentos dentro y fuera de la I.E. Realiza rotación en trabajo y otras funciones afines al cargo que se le asigne.

La señora Rosa Castillo de 35 años, es personal contratado en la I. E. I. N $\mathrm{N}^{\mathrm{o}} 0325$. Es una persona que apoya incondicionalmente a todos los actores educativos de esta institución.

\subsection{Padres de Familia}

La Ley General de Educación 28044, Art.54, considera que los padres de familia, o a quienes hacen sus veces, como tutores, o apoderados que generalmente son los miembros de la familia como hermanos, tíos o abuelos, les corresponde educar a los niños y niñas y proporcionarles en el hogar un trato respetuoso de sus derechos como personas, adecuado para el desarrollo de sus capacidades, y asegurarles la culminación de su educación. Como actores educativos, es su obligación participar y colaborar en el proceso educativo de sus hijos, informarse sobre la calidad del servicio educativo y velar por ella y por el rendimiento académico y el comportamiento de sus hijos.

Las llamadas APAFA son la forma de organizarse en asociaciones de padres de familia. Estos comités u otras instancias de representación tienen como fin contribuir al mejoramiento de los servicios que brinda la correspondiente Institución Educativa. Asimismo, los padres de familia deben apoyar la gestión educativa y colaborar para el mejoramiento de la infraestructura y el equipamiento de la correspondiente Institución Educativa, de acuerdo a sus posibilidades.

Las madres y padres de familia de la I. E. I. No 0325 solicitan continua comunicación con las docentes. Las madres y padres al igual que los niños requieren de las docentes estímulos, atención para que estos se involucren de manera positiva. La diversidad socioeconómica entre ellos es heterogénea: algunos no saben utilizar la tecnología, su economía es variada. La mayoría de los padres son separados y tienen hijos de dos o tres compromisos.

\subsection{Niños y niñas}

En la Ley General de Educación 28044- Art.53, se establece que el estudiante es el centro del proceso y del sistema educativo. Le corresponde un servicio educativo eficiente, con profesores responsables de su aprendizaje y desarrollo integral. Los niños y niñas deben conocer sus derechos y exigir que se cumplan. El respeto entre los actores educativos y el diálogo van en mejora de este servicio que se ofrece a los niños.

La I. E. I. $N^{\circ} 0325$ atiende a niñas y niños de 3 años a 5 años de edad cumplidos. No hay niños con problemas de inclusión. Estas niñas y niños son hijos de padres con distintos problemas, familiares, 
económicos. Entonces, siendo la escuela el primer lugar de socialización. Se fomenta la enseñanza de valores y buenas costumbres. Muchos de sus apoderados son los tíos, abuelos, hermanos mayores, incluso vecinos. Los niños más grandes, de 5 años, tienen problemas de conductas, dicen malas palabras, algunos niños son agresivos.

\section{Comunicación entre actores}

"La comunicación es un proceso esencial en todo ser humano y hace parte de toda estructura social, de allí su relevancia a nivel interpersonal y laboral" (Sánchez y Ospina, 2014, p. 14). ¿Qué ocurre cuando la comunicación falla? Se originan conflictos, no funcionan los servicios con eficacia, se genera tensión entre los actores. Mantener los canales de comunicación es importante para que la institución educativa funcione exitosamente. Se requiere que los actores del proceso educativo asuman una comunicación horizontal que busque lograr los objetivos principales de la institución acorde a los lineamientos del Ministerio de Educación que es la formación de los niños y niñas.

Lo común es culpar al otro de los problemas de la institución. También existe la tendencia a sobresalir entre los demás. Sin embargo, reconocer que los conflictos son una oportunidad para el aprendizaje es fundamental entre los actores, quienes son de distintas esferas culturales y etáreas. En ese sentido, es imprescindible que los actores educativos profundicen en las relaciones que mantienen, en el origen de los conflictos; de modo tal que puedan comprender mejor como solucionarlos y enfocarse solamente en los objetivos de la institución y los logros de los niños y niñas (Minedu, 2013).

La actitud de los actores puede ser el origen de conflictos. la actitud de superioridad, de desconfianza, de desinterés. La actitud hacia el tema que se trata o mensaje y cómo hacerlo llegar pueden ocasionar conflictos. La habilidad para comunicarse se refiere a la capacidad para transmitir o modificar los mensajes que expresen una intención. Es necesaria para esto una competencia sociolingüística que destaque el dominio del lenguaje, la habilidad verbal, corporal y escrita del emisor para hablar, actuar y escribir. No todos los actores tienen esta competencia, menos cuando sus características socioculturales son distintas, empezando por la edad.

Las docentes hacen referencia al nivel de conocimiento que posee el emisor, en este caso, los padres de familia. Esto puede ser prejuicioso y afectar la conducta en el proceso comunicacional. Finalmente, todos los actores tienen un mismo objetivo y debe haber canales y formas de comunicación que posibiliten esto. Llenarse de prejuicios y estereotipos solo perjudica conseguir los fines propuestos. La diferencia sociocultural entre docentes y padres principalmente no debe condicionar los roles que desempeñan cada uno.

La institución debe fomentar una comunicación asertiva y ello se logra con un ambiente laboral respetuoso de las personas y las normas. Finalmente, ese es el principal aprendizaje de los niños. Si los agentes educativos mantienen una comunicación asertiva, lograrán ser más productivos y ofrecer un servicio de mejor calidad, lo cual repercutirá en el sentido de pertenencia de cada actor a la institución. En consecuencia, las actitudes que conlleven una comunicación asertiva fomentarán mejores relaciones sociales y el trabajo en conjunto para lograr las metas propuestas por la institución educativa para el bienestar de los alumnos.

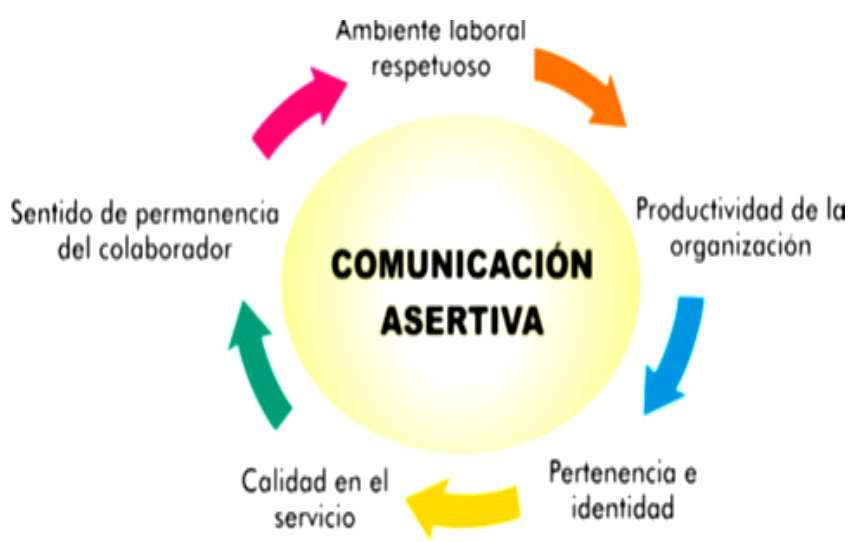

Fuente: Sanchez y Ospina (2014,p. 23)

\subsection{Organización de la institución educativa}

Respecto de su estructura formal, la institución educativa está liderada por la directora del centro. Si bien cada actor tiene conocimiento de su rol, la directora debe velar porque esos roles se cumplan, distribuir roles complementarios, vigilar el desarrollo de los objetivos, uso del tiempo y el espacio. A nivel administrativo, rinde cuentas de los ingresos y egresos económicos de la institución estrategias de gobierno de la institución. Controla la evolución de las acciones. Maneja la información relevante para tomar decisiones.

Respecto de la perspectiva pedagógico-didáctica, todos los docentes deben establecer vínculos 
comunicativos que permitan lograr las capacidades y competencias necesarias en el alumnado. Los actores educativos, principalmente los docentes, tienen un deber con el conocimiento y con su transmisión.

Respecto de las relaciones comunitarias, los actores educativos dentro de la institución deben interesarse por y relacionarse con el ámbito social más amplio, con las familias, con otras instituciones del entorno para afrontar y resolver problemas en común que tienen que ver con el proceso de enseñanza aprendizaje de los niños y niñas.

Ejercer y hacer cumplir estas funciones no debe llevar a un autoritarismo dentro de la institución, es decir, no deben ejercerse funciones jerárquicas que verticalicen la comunicación. La comunicación es una de las principales herramientas de las que disponen los actores educativos y no solo implica hablar, transmitir, sino escuchar.

En las primeras reuniones del año escolar 2018, los padres de familia de la I. E. I. $N^{\text {o }} 0325$ se han mostrado optimistas y deseosos de trabajar en conjunto para cambiar la imagen social que se tiene de la Institución: desde el nombre con que es conocido "Las Totoritas" hasta mejorar la infraestructura y mejorar los canales de comunicación con las profesoras. Sin embargo, debemos asumir un mea culpa a las señales que las profesoras no nos hemos organizado como es debido, no se están ejerciendo los liderazgos adecuados dentro de la institución y la comunicación en lugar de ser asertiva se está volviendo jerárquica.

El principal problema ha sido el doble cargo que asume la directora. Los primeros meses implican organización y trámite administrativo que la aleja del aula y hace que su trabajo sea visto como deficiente por los padres quienes exigen para sus hijos toda la atención de su profesora. Además, el balance económico ha generado confrontaciones con el CONEI quienes no han encontrado satisfactoria la rendición de cuentas por parte de la directora.

Lo cierto es que los padres están cada día más involucrados en el proceso de aprendizaje de sus hijos, lo cual no ocurría de forma masiva el año 2017. En ese sentido, se observa un cambio positivo en la actitud de los padres de familia, pero falta mejorar los canales de comunicación. A diferencia del año 2017, ahora los padres siguen los consejos de las profesoras y cuidan la limpieza de los materiales educativos dentro y fuera del aula, participan de las actividades de limpieza y mantenimiento de la institución. De esta manera, se pretende crear conciencia de pertenencia a la institución.

\section{PEI. Misión de la educación inicial}

La Educación Inicial es el primer nivel del sistema educativo peruano encargado de garantizar una educación de calidad para los niños y niñas de 0 a 5 años de edad. Este nivel crea además las condiciones para generar una cultura de crianza que optimice la función educadora de la familia y comunidad, y articula su acción con la Educación Primaria. Como tal, inicia la formación de competencias previstas en la presente Estructura Curricular y forma estructura cognitiva y afectiva sólida que se constituye en la base del desarrollo de niños y niñas. De esta manera, afirma su compromiso de proporcionar una educación básica y de calidad a todos los niños y niñas del país.

\section{Visión de la I.E. I. No 0325}

Busca ser un centro integral de aprendizaje a través de un innovador proceso de enseñanza, donde se facilite el desarrollo de las capacidades de los niños y niñas, son atendidos por una Educación Inicial oportuna, integral y de calidad, que promueve el desarrollo óptimo de sus potencialidades y responde a la diversidad sociocultural del país. Buscamos conformar un equipo docente y de apoyo, que, para lograr la misión de la escuela, esté comprometido e involucrado de tal manera, que además de atribuirse un alto nivel de profesionalismo, esté profundamente interesado en la felicidad presente y futura de los niños, formando líderes creativos, reflexivos, comunicativos y con visión emprendedora para el éxito, así como su capacidad para transformar su entorno en beneficio de todos. Nuestra línea axiológica se caracteriza por el trabajo eficiente y responsable, teniendo como lema "JUGANDO SÍ SE APRENDE", con los valores de: Puntualidad, Respeto, Solidaridad.

\subsection{Actividades que fomentan las relaciones entre actores}

Existen muchas actividades que deberían fomentar una comunicación asertiva en la escuela. Debemos poner énfasis en estas actividades para que los actores educativos fortalezcan sus relaciones. Las fiestas, por ejemplo, son organizadas por comités de aulas, cuyos miembros trabajan con cada docente. Durante el año escolar se realizan al menos cinco 
fiestas importantes: por el buen inicio del año escolar, para celebrar el Día de la Educación Inicial, cada 24 de mayo (las celebraciones duran toda una semana); el Día del niño se celebra la segunda semana de agosto, el aniversario de la institución se celebra a fines de octubre con paseo de antorchas y cena compartir con la comunidad educativa. Finalmente, la Navidad.

Por otro lado, se organiza a los padres de familia para formar diversas comisiones que deben trabajar de forma articulada durante todo el año escolar. El CONEI es la institución más importante, pero existen otras comisiones: para el Plan de Gestión del Riesgo de Desastres, el Plan Lector, el Plan de Tutoría, para el Reglamento Interno, y los documentos de gestión como el PEI, PCI y PAT. Si bien los padres asumen funciones y responsabilidades, con el paso de los meses las comisiones se desarticulan o se convierten en espacios de conflicto.

El inicio del año escolar 2018 ha estado motivado por una actitud positiva respecto del trabajo en equipo entre los actores educativos. Como resultado de esa disposición se ha llegado a los siguientes acuerdos:

a) Cambiar el nombre de la institución educativa. Hasta el año 2017, desde hace 40 años, se le conoce a nuestra institución con el nombre de Las Totoritas 325 colegio inicial. Ya se ha explicado que este nombre connota la violencia y marginalidad del sector donde queda ubicado el centro educativo. Por eso, se ha propuesto cambiar el nombre a "Emilia y Victoria Barcia Boniffatti" por ser las fundadoras y creadoras del primer Jardín de Infantes en el Perú.

b) Deserción escolar. Es un problema que padece el centro desde el año 2009. Hemos detectado muchos factores como familias desintegradas, cambios de domicilio, cambios de institución educativa. Todavía no sabemos cómo afrontar el problema, pero hay una disposición de las docentes para autoevaluarse y ver los aspectos disfuncionales de la comunicación con los padres de familia las expectativas que estos tienen de la institución. A este problema se une el ausentismo escolar y la promoción educativa.

c) Proyecto de creación de escuela para padres. Es una necesidad imperiosa que creemos fortalecerá la comunicación entre actores y se convertirá en el escenario perfecto para consolidad la comunicación asertiva y resolver conflictos.
Nuestro objetivo es utilizar las 6 Rubricas con las que fuimos evaluadas para no solo mejorar la institución sino ver el resultado que involucra al niño con la participación de su familia y familia escolar porque es la mejor manera que el niño desarrolle sus capacidades tanto cognitivas como emocionales que es la principal idea de una mejor formación para el futuro del ser humano. La idea es involucrar a los padres de familia, aunque tal vez no lo hacemos debidamente porque nos enfocamos demasiado en los niños y niñas. Se debe maximizar la hora de sesión de aprendizaje, buscar estrategias para hacerlos pensar al niño ya no darle lo fácil, mantener los materiales y el aula limpio con ayuda de nuestros niños, padres y todos los actores. Nuestra comunicación debe ser más fluida, la auxiliar está más comprometida en jugar, atender mejor a los niños de acuerdo a lo aprendido con la docente. La idea es que, a través de una comunicación asertiva, los padres valores nuestros esfuerzos.

\section{Informe final de los 5 compromisos del 2017}

1. Progreso anual de aprendizajes de todas y todos los estudiantes de la I.E.

Logros: La mayoría de los estudiantes lograron satisfactoriamente en el desarrollo de su aprendizaje, habiendo obtenido:

\begin{tabular}{lcccccc}
\hline \multirow{2}{*}{ AREAS } & \multicolumn{3}{c}{ INICIO } & \multicolumn{3}{c}{ FINAL } \\
\cline { 2 - 7 } & A & B & C & A & B & C \\
\hline Personal & $\mathbf{2}$ & 7 & $\mathbf{3}$ & $\mathbf{1 0}$ & $\mathbf{1}$ & $\mathbf{1}$ \\
Matemática & - & $\mathbf{5}$ & 7 & $\mathbf{1 0}$ & $\mathbf{1}$ & $\mathbf{1}$ \\
Ciencia y Ambiente & $\mathbf{1}$ & $\mathbf{6}$ & $\mathbf{5}$ & $\mathbf{1 0}$ & $\mathbf{1}$ & $\mathbf{1}$ \\
Comunicación & $\mathbf{2}$ & $\mathbf{5}$ & $\mathbf{5}$ & $\mathbf{9}$ & $\mathbf{2}$ & $\mathbf{1}$ \\
\hline
\end{tabular}

Dificultad: Inasistencia de algunos niños y tardanza en la entrada y salida las cuales fomentaban desorden en el proceso de aprendizaje dificultando el adecuado desarrollo de la clase.

Sugerencias: Seguir concientizando a los padres con diversas estrategias de la importancia para su vida futura el formar el hábito de la puntualidad.

Darles tareas, responsabilidades a aquellos padres que por reiteradas veces siguen llegando tarde.

\section{Retención anual de estudiantes en la I.E..}

Logros: Se realizaron visitas domiciliarias de niños ausentes logrando en un 100\% la retención de los niños durante el año escolar.

Se mantenía informados sobre "La importancia de estudiar el Nivel Inicial" realizando 2 Talleres con padres. 
Dificultades: Se realizaron Talleres de Información y hubo poca asistencia de padres de familia o apoderados. Inasistencia de los niños al inicio de días festivos o feriados largos.

Sugerencias: Continuar con la difusión de la importancia del Nivel Inicial, seguir concientizando a los padres de familia sobre la importancia de la asistencia permanente para un debido proceso de aprendizaje.

3.Acompañamiento y monitoreo a la práctica pedagógica en la I.E.

Logros: Acompañamiento de círculo de aprendizaje

Dificultades: Directora con aula a cargo.

Sugerencias: Habilitar un día para que la Directora nos monitoree y tener un acompañamiento solicitado por la UGEL para una mejora de trabajo.

\section{Gestión de la convivencia escolar en la I.E.}

Logros: Buena convivencia escolar a pesar de dificultades por inasistencia justificadas.

Dificultades: Cuando son apoderados y no asumen la responsabilidad de los padres para poder corregir algún desorden.

Sugerencias: Colocar en los patios las Normas de convivencia del tamaño apropiado que sea visible para la Comunidad Educativa.

\section{CONCLUSIONES}

Las convergencias y divergencias que existen en la comunicación de los actores educativos de la I. E. I. $\mathrm{N}^{\circ} 0325$ generan inestabilidad en el centro respecto de su futuro y continuidad al servicio de la comunidad. Recién el último año y por prerrogativa de la especialista de la UGEL se ha mostrado preocupación por intervenir en la calidad de las interacciones de los actores del sistema educativo, como condicionante para una gestión de calidad y hacia la consecución de los deseados resultados de aprendizaje.

Debe considerarse el factor humano como variable de las divergencias comunicativas: el agotamiento, malestar, problemas personales y estrés docente, hacen que los padres perciban que el clima relacional en la institución educativa se ha deteriorado, así como que las constantes tensiones con la dirección del centro son argumentos para la desacreditación y desconfianza entre actores educativos.
El trabajo entre actores debe ser reflexivo partiendo de la tolerancia y el respeto mutuo. La mejora del clima escolar permitirá lograr los objetivos trazados por la institución. Considérese que esta mejora solo se dará a partir de una comunicación asertiva y horizontal.

Los nuevos padres de familia se ven con fuerza y ganas de grandes cambios, como el de ponerle un nombre nuevo a la institución, pintarlo, embellecer al colegio. Todo esto se debe a la buena Promoción Escolar que se estamos dirigiendo gracias a la evaluación del año 2017 y nos sentirnos capaces de mejorar día a día e impartir los conocimientos que seguimos adquiriendo con las Capacitaciones que recibimos. Sin embargo, esa motivación y ánimo inicial con que se suele iniciar el año escolar debe mantenerse. Ahí radica nuestro trabajo como docentes y el trabajo de la directora como líder de la institución.

Nosotros como Institución queremos que no se vea ese abandono de la infancia que se viene dando en muchas instituciones públicas y privadas, al no aprovechar sus primeros años como formación de mejora para el niño. Nuestra labor como docentes se compara con una casa recién construida. ¿De qué vale tener una casa hermosa, grande? $\mathrm{Si}$ ante cualquier sismo inclusive uno leve se puede derrumbar porque no tiene los cimientos debidos (lo hicieron por hacer) y no le colocaron lo básico la profundidad debida que servirá de sostén para un futuro, para que no se derrumbe, es lo mismo en educación inicial, el niño debe tener una buena educación practica de valores, hábitos, el padre no sabe hacerlo solo por eso necesita la ayuda respectiva de un buen inicio para no incurrir en errores más adelante y tener lamentaciones. 


\section{REFERENCIA BIBLIOGRÁFICA}

Congreso de la Republica (2003) "Ley General de Educación, Ley N²8044, Art.53, 54" Lima, Perú.

Ministerio de Educación (2013) “Aprendiendo a resolver conflictos en las Instituciones Educativas". Lima, Perú.

Murillo, Paulino y Becerra, Sandra. (Septiembrediciembre, 2009). Las percepciones del clima escolar por directivos, docentes y alumnado mediante el empleo de «redes semánticas naturales». Su importancia en la gestión de los centros educativos. Revista de Educación, 350. p p . $375-399$. R e c u p e r a d o d e http://www.revistaeducacion.mec.es/re350/re35 $0 \_16 . p d f$
Sánchez, K., Ospina, Y. (2014). La comunicación asertiva como función integradora de la práctica gerencial. Colección Académica de Ciencias Sociales 1(1), pp. 13-39. Recuperado de https://revistas.upb.edu.co/index.php/cienciasso ciales/article/view/2882 